\title{
Transumbilical endoscopic technique for complete closure of inguinal hernias in female pediatric patients
}

\author{
XUEWU ZHOU ${ }^{1}, \mathrm{XI} \mathrm{QI}^{1}$, BING JIANG ${ }^{1}$, YONGLIANG SHA ${ }^{1}$ and DAIQIANG SONG ${ }^{2}$ \\ ${ }^{1}$ Department of Pediatric Surgery, Xuzhou Central Hospital, Xuzhou, Jiangsu 221009; \\ ${ }^{2}$ Department of Pediatric Surgery, Taizhou Hospital, Taizhou, Zhejiang 317000, P.R. China
}

Received February 8, 2016; Accepted August 23, 2016

DOI: 10.3892/etm.2016.3919

\begin{abstract}
Laparoscopic percutaneous extraperitoneal closure of inguinal hernia with variable devices as an effective technique in minimal-access surgery for pediatric inguinal hernias in children was investigated in the present study. A Veress needle was inserted into the abdomen. Trocars, laparoscope and needle holder were placed through the umbilical incision. Under laparoscopic monitoring, a round needle was introduced into the peritoneal cavity through the anterior abdominal wall. The end of the suture inside the peritoneal cavity was left outside the peritoneal cavity in favor of ligation. The needle was passed through the peritoneum to place an extraperitoneal purse-string suture around the internal inguinal ring, counterclockwise on the left and clockwise on the right. The needle was then passed back into the peritoneal cavity and out through the abdominal wall, and the needle ends of the stitch were cut. The ends of the suture were tied. We described a technique used for transumbilical endoscopic closure of pediatric inguinal hernias in girls. This technique is simple and can be performed quickly. The cosmetic result is excellent because the two umbilical incisions are only 5-mm long, which make it possible to perform surgery without a visible scar. We performed this procedure in over 96 patients and have had no recurrences. Thus, this method may become the laparoscopic repair of choice for females with inguinal hernias.
\end{abstract}

\section{Introduction}

Laparoscopic inguinal hernia repair is now used as an alternative to conventional open herniotomy in children. Although there is ongoing debate regarding the preferred method of inguinal hernia repair, the laparoscopic approach is gaining

Correspondence to: Dr Xuewu Zhou, Department of Pediatric Surgery, Xuzhou Central Hospital, 199 The Liberation of South Road, Xuzhou, Jiangsu 221009, P.R. China

E-mail:nz901n@163.com

Key words: inguinal hernia repair, laparoscopy, children, transumbilical endoscopic surgery popularity because of the potential advantages of faster recovery, attenuated pain, improved cosmetics, and low recurrence rates $(1,2)$. Among various laparoscopic techniques, laparoscopic percutaneous extraperitoneal closure (LPEC) of the hernia is a recently well-developed technique (3). A previous study evaluated the safety, efficacy, and reliability of LPEC in children (4). However, because this technique involves percutaneous closure of inguinal hernias, the inclusion of tissues between the skin and hernial sac, including nerves and muscles, may cause injury and increase the postoperative morbidity in the long-term (5).

The present study described a novel and effective technique to close inguinal hernias with transumbilical endoscopic surgery in girls.

\section{Materials and methods}

The surgical technique was modified according to our previous study (5). Under general anesthesia, patients were laid supine in the Trendelenburg position at a $15^{\circ}$ tilt. The monitor was placed at the patient's feet. The surgeon performed the operation standing on the patient's left side, and the camera assistant stood on the right side. Two 3- or 5-mm curvilinear intraumbilical skin incisions were made. A Veress needle was inserted into the abdomen, and pneumoperitoneum was established at 8 to $12 \mathrm{mmHg}$. One $3-\mathrm{mm}$ trocar and one $5-\mathrm{mm}$ trocar were inserted through the umbilicus, and a $4.5-\mathrm{mm} 0^{\circ}$ laparoscope (Karl Storz GmbH \& Co., Tuttlingen, Germany) and a 3-mm needle holder were placed through the umbilical incision (Fig. 1).

The needle holder was placed on the identical side for the convenience of suturing. Under laparoscopic monitoring, a round needle with 2-0 non-absorbable suture material was introduced into the peritoneal cavity through the anterior abdominal wall near the internal inguinal ring. The suture inside the peritoneal cavity was 5 to $8 \mathrm{~cm}$ long, and the end of the suture was left outside the peritoneal cavity in favor of ligation. The needle was passed through the peritoneum to place an extraperitoneal purse-string suture around the internal inguinal ring (Fig. 2), counterclockwise on the left and clockwise on the right. The needle was then passed back into the peritoneal cavity and out through the abdominal wall, and the needle ends of the stitch were cut. The ends of the suture were tied intraperitoneally with a one-hand tie. 


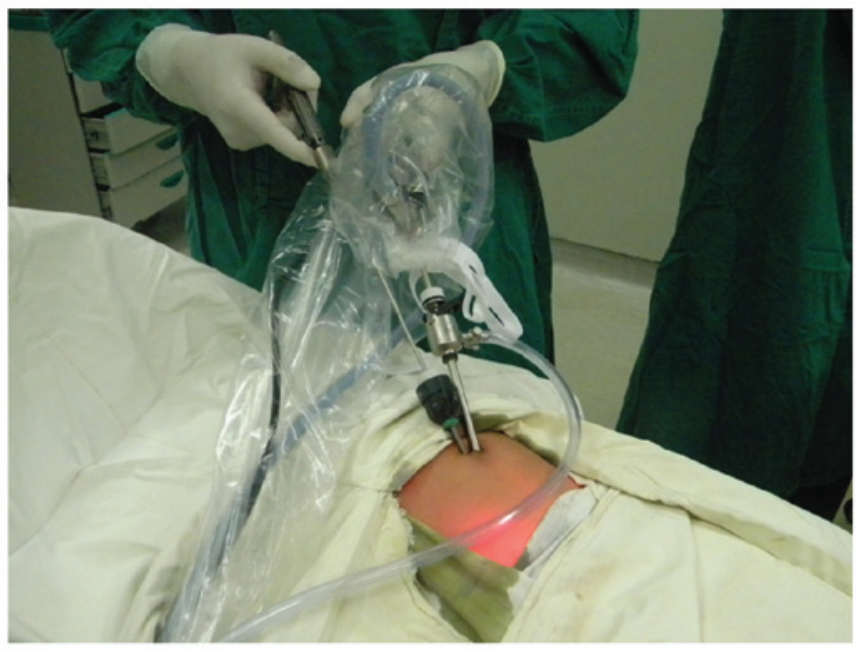

Figure 1. One 3-mm and one 5-mm trocar were inserted through the umbilicus.

The operating assistant maintained tension on the end of the suture outside the abdominal cavity, and a 3- or 5-mm needle holder was used to manipulate the end of the suture inside the peritoneal cavity (Fig. 3). Before the knot of the purse-string stitch was tied, the hernia sac was compressed to expel the gas and liquid within the sac. A triple knot was then performed. Airtightness was confirmed by the absence of hernial sac enlargement when the intraperitoneal pressure was increased. The same procedure was performed on the contralateral side if the processus vaginalis was patent. No stitching was required for the needle and trocar puncture wound. The operative port sites were covered with sterile aseptic absorbent gauze and not sutured. A total of 96 female pediatric patients (age range, 8 months to 8 years; median, 3.4 years) with inguinal hernias ( 25 left-sided, 53 right-sided and 18 bilateral) were included in this study.

\section{Results}

From December, 2009 to August, 2015, 114 procedures were performed in 96 female pediatric patients using the transumbilical laparoscopic intraperitoneal closure technique. The mean operating time was $15 \mathrm{~min}$ (range, 10-20 min) for the unilateral and $24 \mathrm{~min}$ (range, 18-28 min) for the bilateral lesions. All the operations were carried out by senior registrars. The patients did well during the procedure. The findings showed less operative bleeding, no major complications, and no secondary injury for all the groups. The postoperative course was uneventful for all the patients, and no severe perioperative complications were observed. All 96 patients were admitted for day surgery and discharged the same day. The mean follow-up period was 28 months (range, 6-74 months). There were no cases of recurrence or postoperative hydrocele.

\section{Discussion}

Inguinal hernia repair is the most frequently performed procedure in children. Conventional open hernia repair is still the gold standard for children. Laparoscopic inguinal hernia repair in children has recently become an alternative to conventional open herniotomy. Various single-incision laparoscopic surgeries have been reported (5-7). However, given the fact that laparoscopic inguinal hernia repair
A
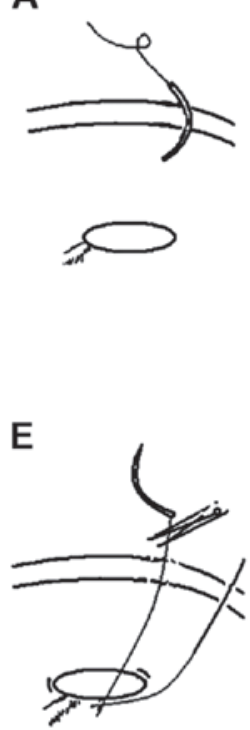

B

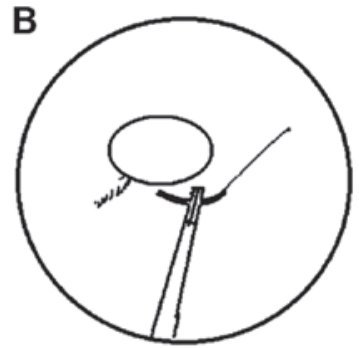

F

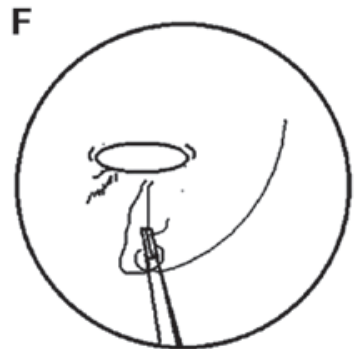

C
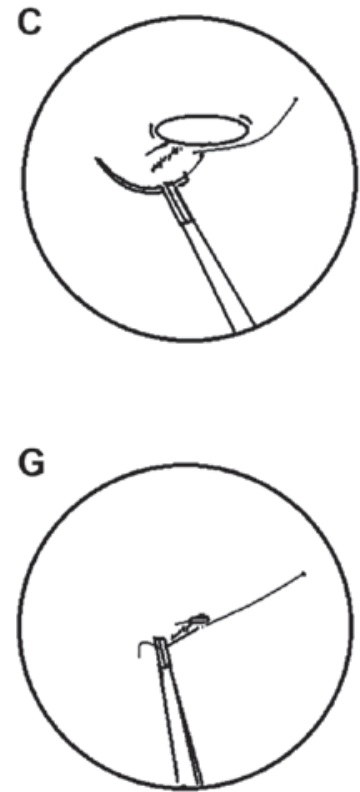

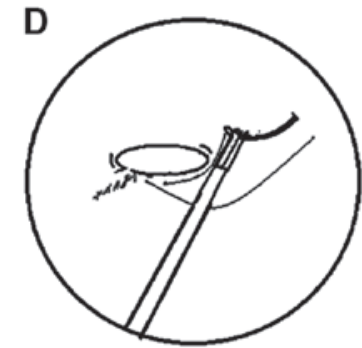

$\mathrm{H}$

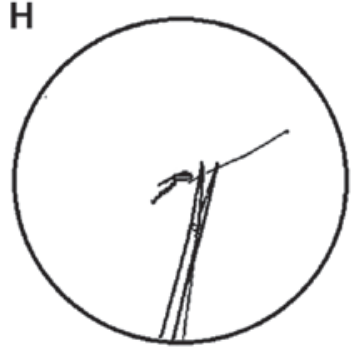

Figure 2. (A) A round needle with 2-0 non-absorbable suture material was introduced into the abdominal cavity near the internal inguinal ring. (B) The needle was passed through the peritoneum to place an extraperitoneal purse-string suture around the internal inguinal ring. (C) The hernial sac orifice was closed extraperitoneally by a purse-string suture around the internal inguinal ring using a round needle. (D) The needle was retrieved through the anterior abdominal wall. (E) The needle was removed after the needle ends of the sutures were cut. (F) The ends of the suture were tied intraperitoneally with a one-hand tie. (G) The operating assistant maintained tension on the end of the suture outside the abdominal cavity, and a 3- or 5-mm needle holder was used to manipulate the end of the suture inside the peritoneal cavity. $(\mathrm{H})$ A triple knot was tied. The ends of the stitch were then cut and were passed out through the abdominal wall. 


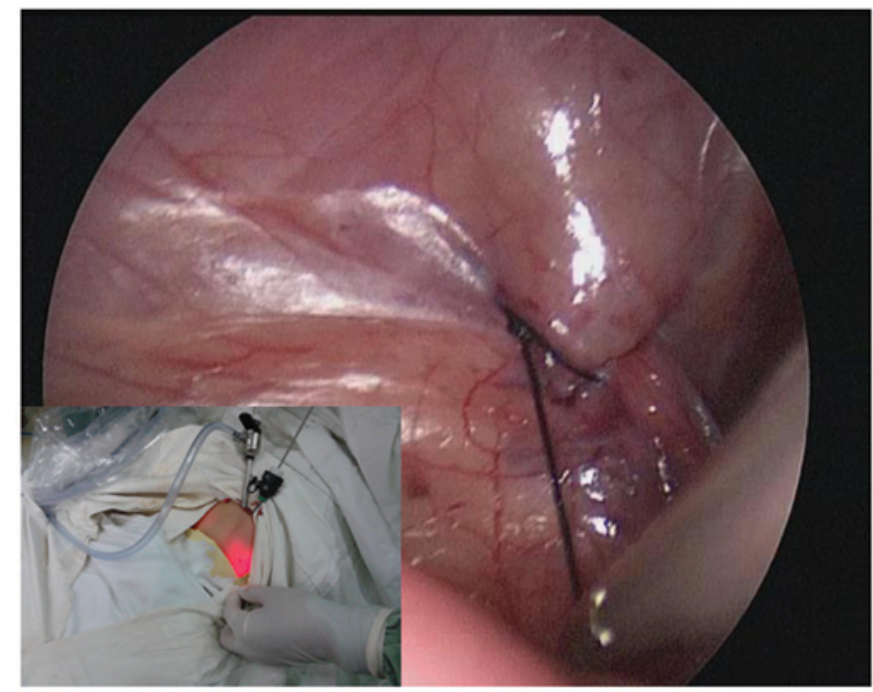

Figure 3. One-hand tie technique for a triple knot.

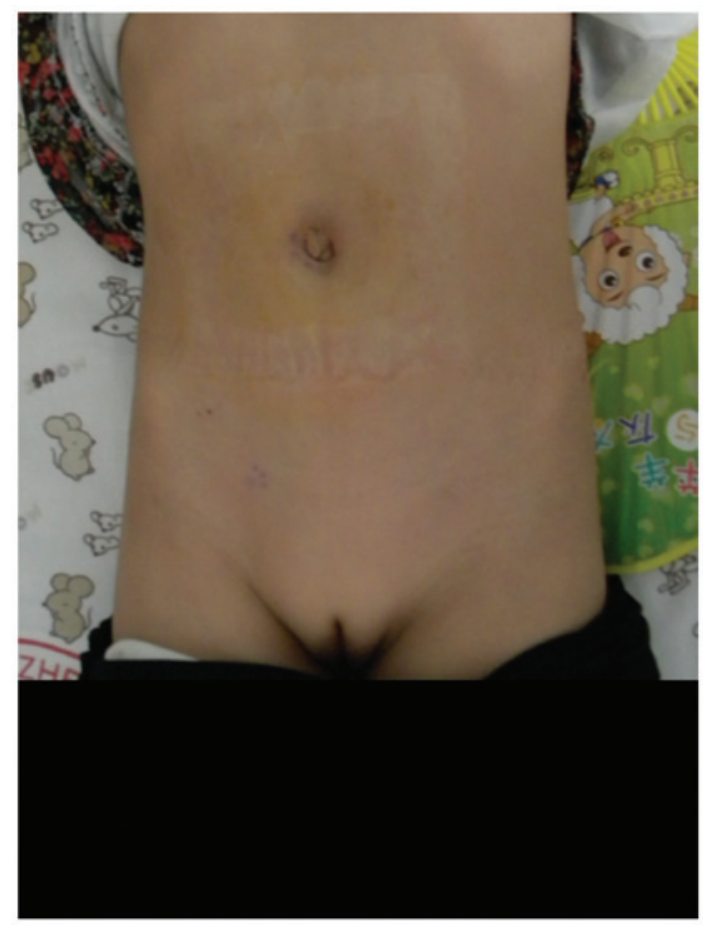

Figure 4. One-day postoperative image of a female patient with bilateral inguinal hernias.

technique involves percutaneous closure of inguinal hernias, tissues between the skin and hernial sac, including nerves and muscles, may be injured by their inclusion in the suture line, which potentially increases postoperative morbidity in the long-term. To preserve the advantages and overcome the limitations of the single-port technique, we designed a novel technique with which to close inguinal hernias with transumbilical laparoscopic intraperitoneal closure in female pediatric patients.

This technique involves transumbilical laparoscopic closure of inguinal hernias without the use of special instruments. It requires one $3-\mathrm{mm}$ trocar and one $5-\mathrm{mm}$ trocar with a $4.5-\mathrm{mm}$ $0^{\circ}$ laparoscope and a $3-\mathrm{mm}$ needle holder. The direction of the purse-string suture is circular, counterclockwise on the left and clockwise on the right to avoid injury to the inferior epigastric artery. Purse-string suturing is easier to perform in female patients because there is no vas deferens or spermatic cord; thus, the operator is less concerned about causing injury. After purse-string suturing, the needle is passed out through the abdominal wall and knots are tied intraperitoneally with a one-hand tie to avoid injury. This technique is simple and can be performed quickly.

Postoperative hernia recurrence is a complication of inguinal hernias. Transumbilical laparoscopic intraperitoneal closure in female patients provides a true high ligation analogous to that performed in the open technique. We observed no recurrence in the patients of the present study during our limited follow-up period.

Scarless endoscopic abdominal surgery has become an important concern in recent years with the development of minimally invasive surgery. A new concept known as natural orifice transluminal endoscopic surgery appeared with the publication of the first experimental study by Kalloo et al (8); however, few clinical studies explaining this method exist in the literature. The major limiting barriers for its clinical application include access, closure, infection, suturing technology, and orientation (9). Compared to natural orifice transluminal endoscopic surgery, transumbilical endoscopic surgery is a less complicated and safer method. Transumbilical endoscopic surgery produce scarless outcome and theoretically has the same advantage of rapid recovery. Undoubtedly, transumbilical endoscopic surgery is an option for scarless abdominal surgery.

Compared to the current single-incision laparoscopic extraperitoneal closure techniques, our method is easy to perform and the instruments are readily available. Intraperitoneal ligation may enclose the internal ring without a peritoneal gap. Our preliminary experience showed satisfactory outcomes with the transumbilical laparoscopic intraperitoneal closure technique for complete endoscopic closure of inguinal hernias in female pediatric patients. Cosmetic result and the ability to simultaneously detect and repair contralateral patent processus vaginalis are the two main advantages of laparoscopic surgery over open surgery. Our results suggest that transumbilical endoscopic surgery for inguinal hernias in female pediatric patients is safe, effective, and reliable.

Two-port transumbilical laparoscopic intraperitoneal closure of inguinal hernias is an effective technique in minimal-access surgery for pediatric patients. The cosmetic results of transumbilical laparoscopic intraperitoneal closure in the present study were excellent because of the presence of only two umbilical wounds for the unilateral and bilateral hernias. The wound scar associated with the laparoscopic port was hidden in the umbilicus, and the puncture wounds made by the devices were minimal. This technique is more suitable for female patients with bilateral hernias (Fig. 4).

In the present study, because the hernia sac was closed intracorporeally, the risk of intra-abdominal complications was not totally eliminated. Although serious complications such as unintentional puncturing of the iliac vein and intra-abdominal viscus did not occur, future studies with greater numbers of patients and long-term follow-ups should be conducted. 


\section{References}

1. Niyogi A, Tahim AS, Sherwood WJ, De Caluwe D, Madden NP, Abel RM, Haddad MJ and Clarke SA: A comparative study examining open inguinal herniotomy with and without hernioscopy to laparoscopic inguinal hernia repair in a pediatric population. Pediatr Surg Int 26: 387-392, 2010.

2. Endo M, Watanabe T, Nakano M, Yoshida F and Ukiyama E: Laparoscopic completely extraperitoneal repair of inguinal hernia in children: a single-institute experience with 1,257 repairs compared with cut-down herniorrhaphy. Surg Endosc 23: 1706-1712, 2009.

3. Li S, Li M, Wong KK, Liu L and Tam PK: Laparoscopically assisted simple suturing obliteration (LASSO) of the internal ring using an epidural needle: a handy single-port laparoscopic herniorrhaphy in children. J Pediatr Surg 49: 1818-1820, 2014.

4. Takehara H, Yakabe S and Kameoka K: Laparoscopic percutaneous extraperitoneal closure for inguinal hernia in children clinical outcome of 972 repairs done in 3 pediatric surgical institutions. J Pediatr Surg 41: 1999-2003, 2006.
5. Chang YT: Technical refinements in single-port laparoscopic surgery of inguinal hernia in infants and children. Diagn Ther Endosc 392847: 1-6, 2010.

6. Wen XQ, Huang WT, Situ J, et al: Single-port laparoscopic radical prostatectomy: initial experience and technical points to reduce its difficulties. Chin Med J 124: 4092-4095, 2011

7. Chang Y-T, Wang J-Y, Lee J-Y and Chiou C-S: A simple single-port laparoscopic-assisted technique for completely enclosing inguinal hernia in children. Am J S 198: e164-e167, 2009.

8. Kalloo AN, Singh VK, Jagannath SB, Niiyama H, Hill SL, Vaughn CA, Magee CA and Kantsevoy SV: Flexible transgastric peritoneoscopy: a novel approach to diagnostic and therapeutic interventions in the peritoneal cavity. Gastrointest Endosc 60: 114-117, 2004.

9. Zhu JF, Hu H, Ma YZ, Xu MZ and Li F: Transumbilical endoscopic surgery: a preliminary clinical report. Surg Endosc 23: 813-817, 2009. 